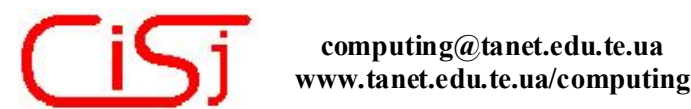

\title{
GMSK NEURAL NETWORK BASED DEMODULATOR
}

\author{
Andrea Aiello (1), Domenico Grimaldi (2), Sergio Rapuano (1)

\begin{abstract}
(1)Facolta di Ingegneria, Universita del Sannio, Piazza Roma, 82100 Benevento, Italy. Ph: ++390824 305817, Fax: ++390824305840, E-mail: \{aiello, rapuano\}@unisannio.it (2)Dip. di Elettronica, Informatica e Sistemistica Universita della Calabria, 87036 Rende (CS), Italy. Ph:++39 0984 494712, Fax: ++39 0984 494713, E-mail: grimaldi@deis.unical.it
\end{abstract}

Abstract: In this paper, the pattern recognition characteristics of the Artificial Neural Networks are used to realise a real demodulator for Gaussian Minimum Shift-Keying signals, used in the GSM telecommunications. The demodulator utilises the Learning Vector Quantisation (LVQ) neural network. It offers both greater efficiency in demodulating and less sensitivity to noise. In order to solve the problem regarding input signal synchronisation, a pre-processing phase is organised. The demodulator prototype has been realised by implementing the pre-processing phase and the LVQ neural network on TMS320C30 Digital Signal Processor. The demodulator has been tested according to the European Telecommunication Standard Institute Recommendations.

Keywords: - Neural network, LVQ, GMSK modulation.

\section{INTRODUCTION}

The Artificial Neural Networks (ANNs) are more efficient than the conventional algorithms and represent an interesting tool for advanced research and applications both in measurement and signal processing $[1,2]$.

In particular the ANNs promise to be more efficient in recognising and distinguishing complex vectors according to their ability to generalise and to form some internal representations of the supplied input signal $[3,4]$.

These abilities make them very useful for the demodulation of the Gaussian Minimum ShiftKeying (GMSK) signals, used in GSM telecommunications.

In the GMSK signal, the information is carried by the phase of the modulated signal. In particular, the slope of the signal depends on the transmitted bit. A positive slope means that a binary " 1 " has been transmitted, while a negative slope means that a binary " 0 " has been transmitted. The GMSK demodulator must extract the phase from the modulated signal and, by using a slope classifier, decode the transmitted bits.

The demodulation of the GMSK signal is very difficult in real cases. In fact, the transmitted signal is corrupted by channel noise, by Doppler effect and by multi-path propagation.

Others difficulties arise from the international recommendations of the European Telecommunication Standard Institute (ETSI) [5]-[7] that establishes the parameter values to be respected by real GMSK demodulator. These parameters are very restrictive and concern both (i) the modulated signal characteristics, and (ii) the demodulation time interval.

Several ANNs could be utilised for GMSK demodulation. A slope classifier based on the Learning Vector Quantisation (LVQ) ANN [8] has been designed and tested. The LVQ neural network shows the greatest benefit with regard to the characteristics of simplicity and performance [9].

This demodulator does not require recovery of the carrier phase and frequency. According to the classical non-coherent demodulation schemes, it performs a bit-per-bit demodulation and does not use the memory of the modulation process [10].

The prototype of the neural-based GMSK demodulator has been realised by implementing the LVQ neural network on a Digital Signal Processor (DSP). This prototype permits to test the demodulator performance according to the international recommendations.

In the following, the basics of GMSK signals are briefly illustrated. Then, the proposed neural based GMSK demodulator is described in detail. Its performance is assessed by means of some experimental tests. The test results, according to the international recommendations, are given in order to evaluate the performance of this neural demodulator versus the Signal to Noise Ratio (SNR).

\section{GMSK SIGNAL IN BRIEF}

GMSK modulation has been widely used in several wireless telecommunication systems, i.e. for GSM cellular telephone system in Europe, for 
CDPD (Cellular Digital Packet Data) system in the USA.

The GMSK modulation belongs directly from continuous-phase Frequency Shift Keying (FSK) with a modulation index $(\mathrm{h}=0.5)$ that will produce orthogonal signalling.

In the GMSK modulation, the phase modulated signal is

$$
s(t)=S_{M} \cos \left[2 p f_{c} t+j_{t}(t)\right]
$$

where $f_{c}$ is the carrier frequency and $j_{t}(t)$ is the information carrying phase.

In the case of multi-path propagation, Doppler and fading effects, according to the ETSI international recommendations [5]-[7] the model of the signal to be taken into account is

$$
s(t)=e_{n} c_{n} \cos \left(2 p f_{c} t+j_{t}(t)+j_{n}+2 p D f_{n} t\right)
$$

where: Дf $\mathrm{f}_{\mathrm{n}}= \pm \frac{\mathrm{v}}{\pi} \cos \sigma_{\mathrm{n}}$, with $\mathrm{v}$ the speed of mobile station and 1 the signal wavelength, $\mathrm{j}_{\mathrm{n}}$ the phase uniformly distributed in the range $[0,2 p], c_{n}$ the signal amplitude evaluated according to the Rayleigh distribution [6].

In ideal situation a simple slope classifier can decode the modulate signal easily and quickly (Fig.1). In real situation the transmitted signal is corrupted by channel noise, by Doppler and multipath effects so that the bit decoding is not easy. Fig. 2 a) and b) show the modulated signal without noise, Fig2 c) and d) the same signal with Additive White Gaussian Noise (AWGN). In order to realize a very reliable GMSK decoder a slope classifier based on the neural network has been designed and tested.

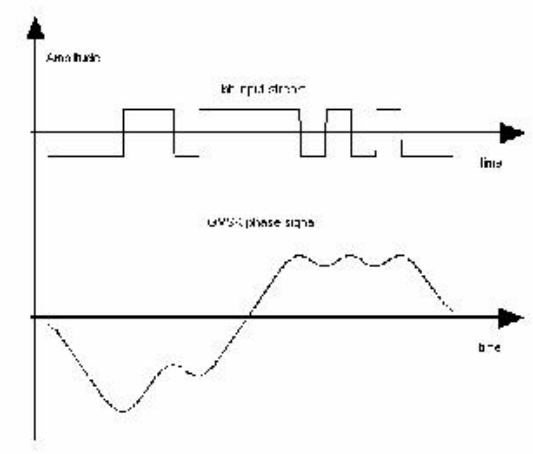

\section{Fig.1 GMSK phase signal and corr esponding bit input stream.}

\section{THE PRE-PROCESSING PHASE}

Before being sent to the neural network demodulator, the phase signal is pre-processed according to the following rules:

1. the baseband phase signal is sampled;

2. synchronisation is performed by detecting the first change to the signal slope;

3. the different phase shifts of the incoming
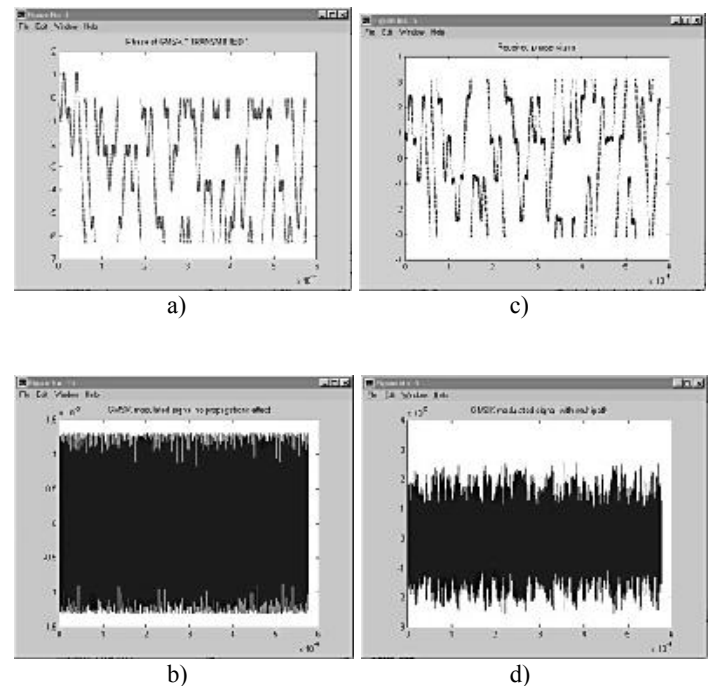

Fig.2 a) and b) the modulated signal without noise, c) and d) the same signal with Additive White Gaussian Noise.

modulated signal are detected and organized to send to the demodulator. This step is shown in Fig.3;

4. 18 samples for each transmitted bit are stored;

5. four samples, selected from the central zone of the 18 samples, are fed into the modulator.

The sampling frequency and the sample number are determined according to the optimisation of the LVQ architecture.

Therefore, the demodulator must perform only the slope classification.

\section{ANN-BASED SLOPE CLASSIFIER}

The GMSK slope classifier is based on the LVQ neural network [8]. The LVQ neural network is able to decode the input signal by classifying the phase's slope which depends on the transmitted bit.

\subsection{The LVQ neural network}

Given $\mathrm{N}$ classes of d-dimensional vectors and a vector $\mathrm{x} \in \mathfrak{R}^{\mathrm{d}}$, the LVQ neural network classifies $x$ by individualising the class to which it belongs. For this purpose, the ANN needs a training phase, in which a reference vector $y$, called the codebook, is determined for each class. Next, the LVQ network classifies a vector individualising the codebook, which best matches it. The used matching function, that shows the better performances, is the Euclidean distance

$$
\mathrm{d}_{\mathrm{e}}(\mathrm{x}, \mathrm{y})=\sum_{\mathrm{j}=1}^{\mathrm{n}}\left(\mathrm{x}_{\mathrm{j}}-\mathrm{y}_{\mathrm{j}}\right)^{2} \text {. }
$$

The neural network is provided by a fragment of the baseband phase signal, it classifies its slope and furnishes the transmitted bit as output. 


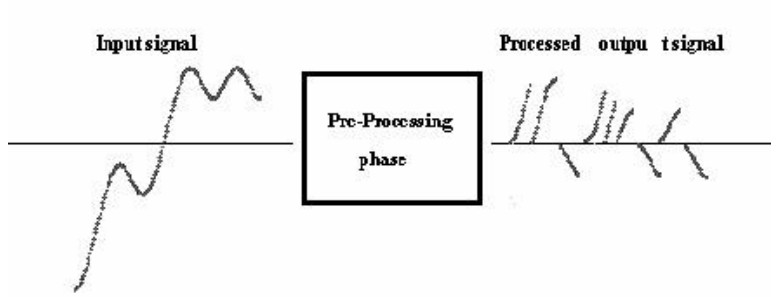

Fig.3 Pre-processing phase to select and extract the different phase shift.

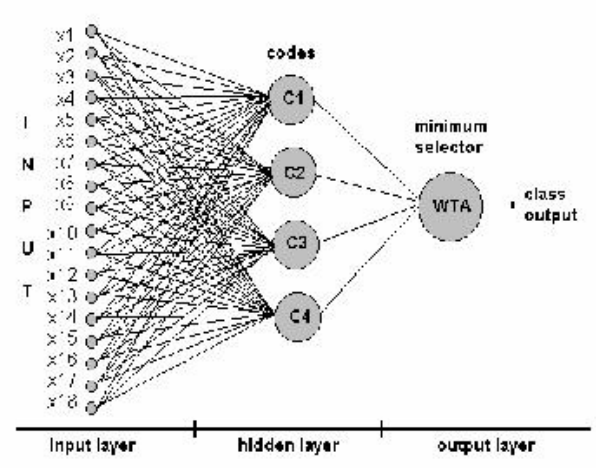

Fig.4 LVQ neural network layout.

The lay-out of the LVQ neural network is shown in Fig.4. The input layer of the LVQ network is connected directly to the hidden layer without weighting the signal. The neurons of the hidden layer calculate the matching function between the input vector and their associated codebook according to (3). Each codebook corresponds to pre-fixed slope. The output layer neuron calculates the winning matching function as being the largest amongst the output of each hidden layer neuron in order to determine the detected slope.

\subsection{The initialisation phase}

The number of both the input neurons and codebooks is set during the initialisation phase.

The number of input neurons depends on (i) satisfying the international recommendations GMSK signal demodulation, and (ii) the sampling frequency of the incoming signal.

By taking into account these problems, and in order to make the demodulator robust, less sensitive to the noise, the sampling frequency of the realised prototype has been set equal to $4.5 \mathrm{MHz}$ so as to obtain 18 samples for symbol when the signal is acquired in the base band. Only 4 samples, selected in the central zone of each symbol, are sent to neural demodulator. In this manner the problems depending on the sample leakage at the end of the symbol are avoided.

The number of the codebook is fixed equal to 4, two for each slope.

Fig.5 show the lay-out of the neural modulator and the criteria to select the 4 samples into the 18 element input vector.

In the output layer, the minimum $d_{e}$ is evaluated and the decoded bit is determined.

\subsection{The training phase}

The training set is constituted by the phase slope of 5 random bursts of a GSM signal for a total of $151 \times 5$ random bits.

The ANN is trained by using the LVQ2 algorithm [8]. This last works as follows: let $\mathrm{x}$ be a vector of the training set and $\mathrm{m}_{\mathrm{w}}$ the winning codebook according to (3). The codebook is updated according to:

$$
m_{w}(t+1)=m_{w}(t) \pm a(t)\left[x-m_{w}(t)\right]
$$

The plus sign is used if $\mathrm{x}$ and $\mathrm{m}_{\mathrm{w}}$ belong to the same class, the minus sign otherwise. This process is repeated for 40-45 iterations for each vector of the training set. The a $(\mathrm{t})$ training coefficient (ranging in 0 to 1 ) is empirically determined. A larger value is usually adopted during the first iterations, while a small one is preferred for the last ones.

The block scheme of the learning phase is shown in Fig.6.

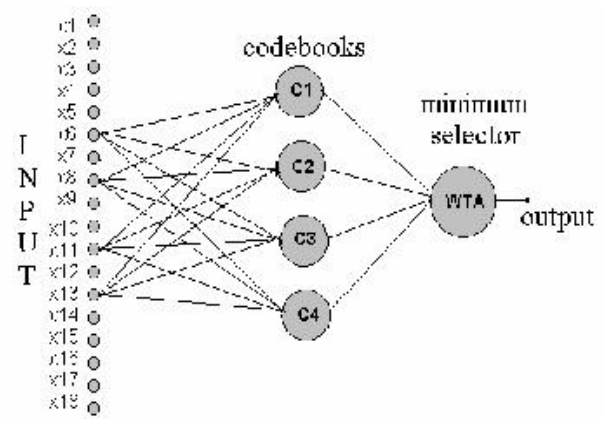

Fig.5 Layout of the LVQ neural network and selection of input samples.

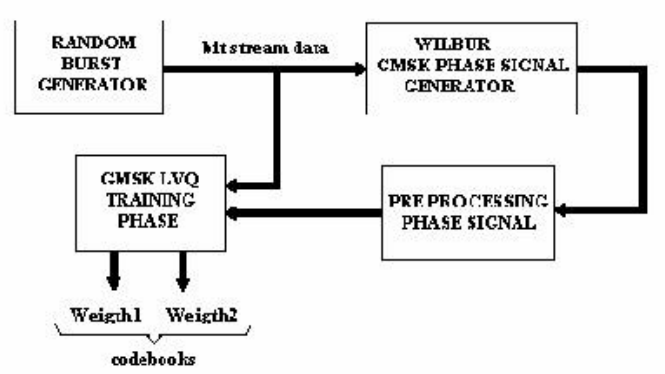

Fig.6 Block scheme of the learning phase.

\section{GMSK DEMODULATOR IMPLEMENTED ON DSP}

Once trained, the pre-processing phase and the LVQ neural network are implemented on the DSP TMS320C30 by Texas Instruments [11]. 
Table 1 Processing time for different LVQ based demodulator

\begin{tabular}{|c|c|c|l|}
\hline \multicolumn{2}{|c|}{ LVQ } & $\begin{array}{l}\text { processing } \\
\text { time }[\mathrm{ms}]\end{array}$ & $\begin{array}{l}\text { ETSI } \\
{[\mathrm{ms}]}\end{array}$ \\
\hline samples & codebooks & 120 & $<5$ \\
\hline 18 & 8 & 50 & $<5$ \\
\hline 18 & 4 & 9.5 & $<5$ \\
\hline 8 & 4 & 4.7 & $<5$ \\
\hline 4 & 4 & \multicolumn{2}{|c}{} \\
\hline
\end{tabular}

The codebooks are recorded on the DSP memory into an array. The use of the array instead of the matrix makes processing more efficient because only one access is needed instead of two accesses required by matrix (Fig.7).

\section{TESTS ACCORDING TO THE ETSI RECOMMENDATION}

The neural demodulator has been tested according to the European Telecommunication Standard Institute (ETSI) recommendation [5]-[7]. In all the tests, GMSK modulated signals with $\mathrm{BT}_{\mathrm{s}}=0.3$, where B is the band width, are used.

In order to execute estimation of the Bit Error Rate (BER), several channel models have been simulated.

The models, as established by the ETSI recommendation [5], are the Rural Area characterised by maximum speed $250 \mathrm{~km} / \mathrm{h}$ (RU 250), Total Urban maximum speed $50 \mathrm{~km} / \mathrm{h}$ (TU 50) and Hilly Terrain maximum speed $100 \mathrm{~km} / \mathrm{h}$ (HT 100). These tests are called dynamic because the receiver is in motion. Moreover, a static test has been carried out in which the channel is assumed to be characterised by Additive White Gaussian Noise (AWGN) and the receiver is motionless.

All the tests have been carried out according to the methods described by the ETSI 300113 recommendation.

The test results are shown in Fig. 8 for LVQ neural networks characterized by both different input samples and codebooks. The results are compared with the values imposed by the recommendation.

Fig.9 gives the BER versus the ratio $E_{b} / N_{0}$, where $\mathrm{E}_{\mathrm{b}}$ is the signal energy per bit, $\mathrm{E}_{\mathrm{b}}=\mathrm{S}_{\mathrm{M}}{ }^{2} \mathrm{~T} / 2$, and $\mathrm{N}_{0}$ the spectral density of the assumed white and Gaussian noise. In the HT area, a performance degradation of the demodulator is observed. The received signal is heavily distorted by the presence of a great number of different paths.

The processing time of each of the four different LVQ based demodulators is reported in Tab.1. Only the LVQ with 4 input samples and 4 codebook satisfy the ETSI recommendation.
Table 2 Percentage of correct demodulation

\begin{tabular}{|c|c|}
\hline \multicolumn{2}{|c|}{ for different bit streams } \\
\hline bit stream & $\begin{array}{c}\text { correct } \\
\text { demodulation } \\
{[\%]}\end{array}$ \\
\hline “010101..." & 99 \\
\hline "111111..." & 98 \\
\hline "000000..." & 98 \\
\hline Burst TCH & 98 \\
\hline Burst TRAIN & 98 \\
\hline
\end{tabular}

The LVQ based demodulator was tested by using real GMSK signals. These signals were characterised by: carrier frequency equal to $21.4 \mathrm{MHz}$, sample rate equal to $200 \mathrm{MS} / \mathrm{s}$.

Five different bursts of signals, shown in Fig. 10, were sent to the neural demodulator. Tests were carried out by sending 100 bursts with the same bit stream.

Tah. 2 shows the nercentage of correct demoduTh: <-lh far ant of tre chase

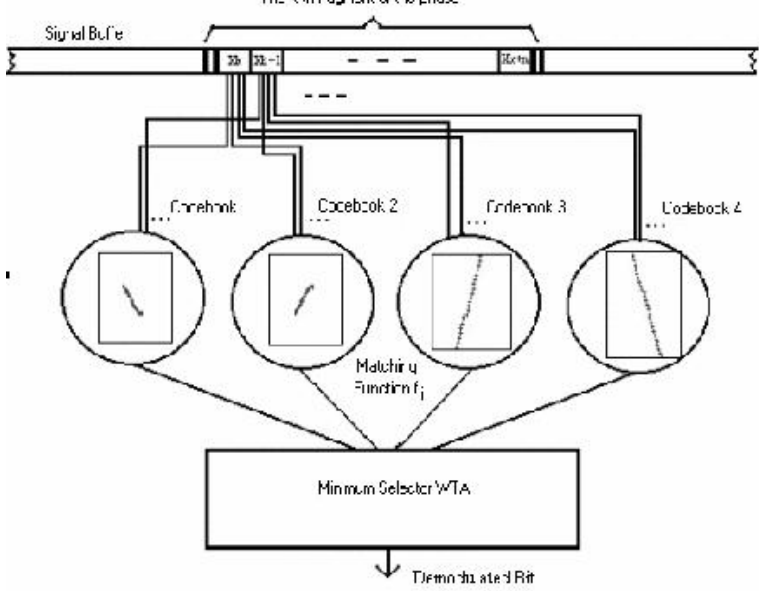

Fig.7 Logical scheme for implement on DSP the LVQ neural network with four codebooks for GMSK signal demodulation.
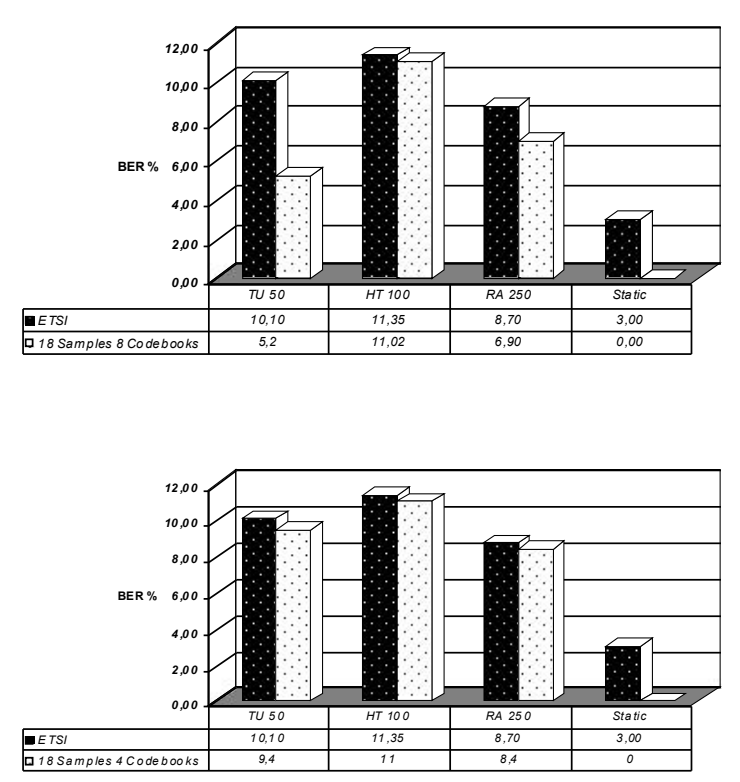

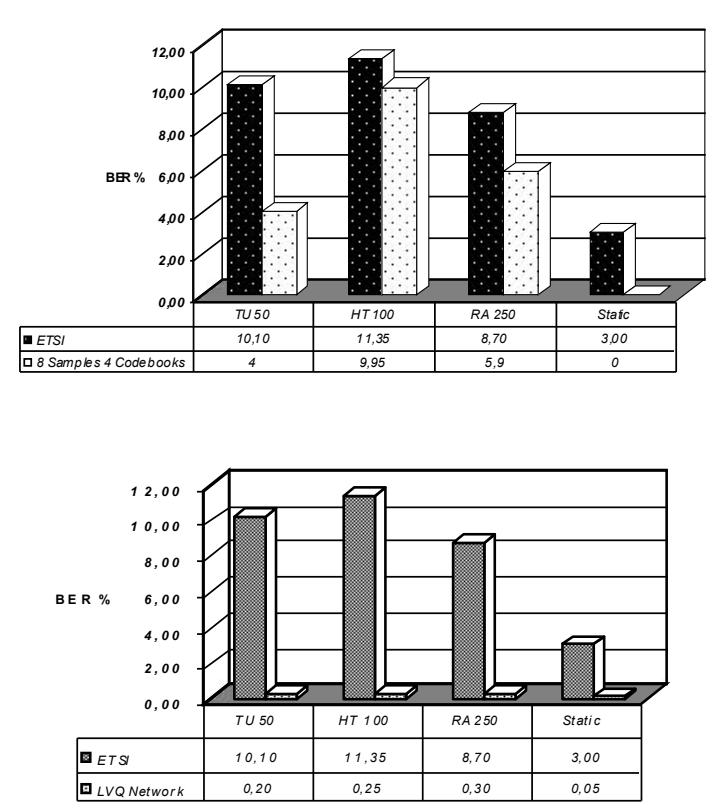

Fig.8 BER for the neural based demodulator compared with ETSI values for different condition of propagation. The bottom refers to 4 input samples and 4 codebooks.

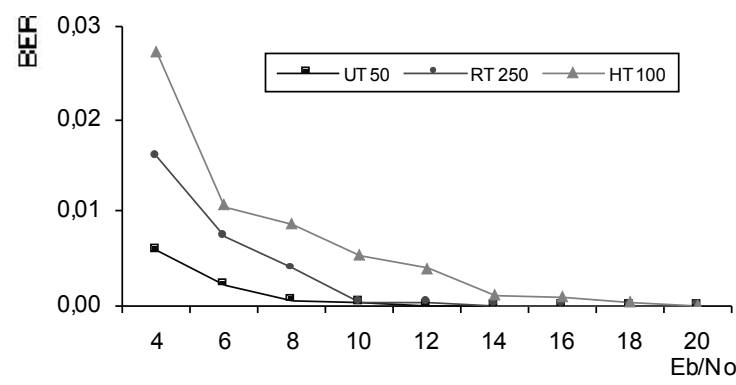

Fig.9 BER for the neural based demodulator for different condition of propagation versus $E_{b} / N_{0}$

The experimental tests confirmed that the demodulator meets the ETSI Recommendation and can be used successfully in real applications.

\section{ACKNOWLEDGMENTS}

The authors wish to thank Prof. P. Daponte for his support, suggestions, and helpful guidance in performing the research.

\section{REFERENCES}

[1] P. Daponte, D. Grimaldi, "Artificial neural networks in measurement", Measurement, vol.23, No.2, March 1998, pp.93-115.

[2] P. Daponte, D. Grimaldi, L. Michaeli, "Analog neural network design for processing of measurement signals", Measurement, vol.26, No.2, March 1999, pp.1-17

[3] P. Daponte, D. Grimaldi, L. Michaeli, "Neu- ralnetwork and DSP based decoder for DTMF signals", IEE Proc. on Science, Measurement and Technology, vol. 147, No. 1, Jan. 2000, pp. 34-40.

[4] A. Aiello, P. Daponte, D. Grimaldi, L. Michaeli, "Artificial neural network for DTMF decoders"; Proc. Of ICNNAI'99, 12-15 October, Brest, Belarus, pp.110-116, 1999.

[5] ETSI/GSM Recommendation 05.05/ETS 300-577, GSM and DCS1800 Digital cellular telecommunication system (Phase 2+) Radio transmission and reception, July 1996.

[6] ETSI/GSM Recommendation 11.10/ETS 300-607, GSM and DCS1800 Mobile station conformance specification. Part 1: Conformance specification, July 1996.

[7] ETSI/GSM Recommendation 11.21/ ETS300-609, Base station system (BSS) equipment specification. Part 1:Radio aspect, July 1996. [2]

[8] T. Kohonen, Self-organizing maps, Springer, 1997.

[9] A. Abrardo, G. Benelli, G.R. Cau, "Multiplesymbol differential detection of GMSK for mobile communications", IEEE Trans. on Vehicular Tech., vol. 44, No.3, August 1995, pp.379-389.

[10] S. M. Redl, M. K. Weber, M. W. Oliphant, An introduction to GSM, Artech House Publ., 1995.

[11] TMS320CX, User's Guide, Texas Instruments, 1994.

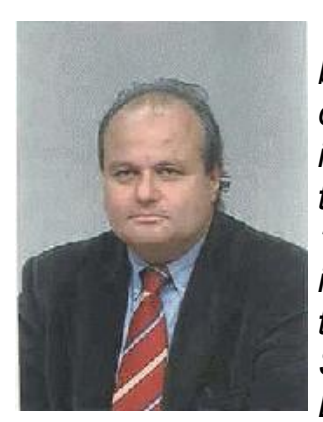

Domenico Grimaldi was born in Italy in 1952. He received the degree "cum laude" in Electrical Engineering from the University of Naples in 1979. He, after working as an independent consultant and teacher in the Technical School until 1990, joined the Department of Electronic, Computers and System Science of the University of Calabria, Italy, as assistant Professor of Electronic Measurements. At the moment it is Associated Professor of Electronic Measurement at the same Department. Mr. Grimaldi has remained there in a variety of research and management positions. From 1999 to 2000 it was responsible of the research unit in the frame of National Project PRIN supported by the University Ministery. From 1997 to 2001 it was contact person in Tempus Project and Leonardo da Vinci Project of the European Union. From 1998 he is delegate of the Rector of the University of Calabria to the degree orientation. In 1997, 1998 and 1999 it was visiting professor at the Technical University of Kosice (Slovak Republic). Mr. Grimaldi is a member of the IEEE Instrum. and Meas. Society, and AEI (the Italian Institute of Electrical Engineers), and from 1998 to 
2000 it was vice-president in the Calabria Region. He has published about 80 papers and his current researches include the characterizasion of measurement transducers, neural modeling for $A D C$ and measuring systems, digital signal processing for monitoring and testing, virtual instrumentation and distributed measurements.

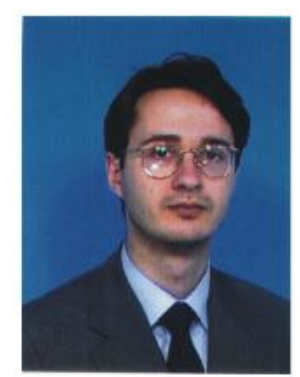

Sergio RAPUANO achieved the master's degree "cum laude" in Electronic Engineering from University of Salerno in 1999. Then, he joined the research activities carried out at the Laboratory of Signal Processing and Measurement Information of the University of Sannio, Benevento. Since 1999 he has been attending a PhD course in Software Engineering, Telecommunications and Applied Electromagnetism. At the present time he is developing his research work in digital signal processing for measurement in telecommunications, data converters, power quality and non-destructive testing. In 2002 he joined the Faculty of Engineering of University of Sannio as Assistant Professor of Electric and Electronic Measurement. 